\title{
Imatinib Induced Tumor Lysis Syndrome in a Patient with Gastrointestinal Stromal Tumor
}

\author{
Hatim Karachiwala MD, FRCPC, ABIM', Bruce Colwell MD, FRCPC, BSc ${ }^{1}$ \\ ${ }^{7}$ Department of Medicine, Divison of Medical Oncology, Dalhousie University
}

\begin{abstract}
Tumor Lysis syndrome (TLS) is a well-known oncological emergency. It is most commonly associated with bulky and highly proliferative tumors such as leukemias and lymphomas where destruction of cells, usually from chemotherapy, leads to hyperuricemia, hyperkalemia, hyperphosphatemia and hypocalcemia. Such metabolic derangements can lead to renal failure and potentially fatal cardiac arrhythmias.

New biologically directed therapies such as imatinib mesylate, a selective tyrosine kinase inhibitor against the proto-oncogene c-kit, may make TLS more common. We present a case of a patient with progressive metastatic gastrointestinal stromal tumor who, during treatment with imatinib mesylate, developed severe TLS ultimately leading to death. This case outlines the importance of clinicians to ensure appropriate monitoring of patient's metabolic status during treatment with cytotoxic chemotherapy and biologically directed therapies, including oral agents such as imatinib, and to consider rasburicase or allopurinol and hydration as prophylaxis in high-risk populations.
\end{abstract}

Tumor lysissyndrome(TLS) is an oncological emergency usually seen with highly proliferative tumors such as leukemias and lymphomas, most often occurring after a good response to chemotherapy. It is rarely seen in solid tumors, except for the rare case of small cell or germ cell tumors. ${ }^{1,2}$ TLS occurs when tumor cells are destroyed and the cell contents are dispersed, causing various metabolic derangements including elevated potassium, phosphorous, and uric acid levels and decreased calcium levels. If not identified promptly and treated appropriately it can lead to acute renal failure, due to precipitation of calcium and phosphorous in kidney tubules. The inability of the kidneys to excrete large amounts of potassium from rapid cell lysis may, in turn, also lead to lethal cardiac arrhythmias.

Gastrointestinal stromal tumors (GISTs) are known to respond rapidly to imatinib mesylate and other c-kit directed tyrosine kinase inhibitors. Prior to the introduction of these medications, these tumors did not respond well to treatment and historically have not been associated with TLS. We present a case of a patient diagnosed with GIST who underwent treatment with imatinib and upon starting treatment developed acute renal failure secondary to TLS from which the patient did not recover and unfortunately died.

\section{Case presentation}

A 60-year-old female presented to our SUNY Upstate Cancer Center for follow-up in early November 2009 following a laparotomy, total abdominal hysterectomy with bilateral salpingo-oopherectomy, omentectomy and excision of a pelvic mass in early June 2009. The pelvic mass was found to be CD117 positive and CD34 negative on immunohistochemical staining and was diagnosed as a GIST. To our knowledge there was no evidence of any residual mass present after surgery. The patient's past medical history included hypertension and hyperlipidemia, but was otherwise unremarkable.

Upon recovery from surgery, the patient was reviewed in clinic and was prescribed imatinib $400 \mathrm{mg}$ daily as adjuvant treatment to decrease her risk of cancer recurrence. However, after three doses she developed severe nausea and vomiting and was admitted to our inpatient facility for management. At this time, the patient also complained of profound fatigue and non-specific abdominal pain. Laboratory investigations revealed no derangements in kidney function (serum creatinine $79.2 \mathrm{umol} / \mathrm{L}$ ) or electrolytes (serum calcium $2.4 \mathrm{mmol} / \mathrm{L}$, potassium $4.4 \mathrm{mmol} / \mathrm{L}$ ). Uric acid and phosphorous levels were not initially checked on admission. An abdominal computed tomography scan (CT) with contrast was done, which showed a new large mass impinging on the patient's stomach and pancreas. A diagnosis of partial small bowel obstruction secondary to tumor recurrence was made. The patient was treated conservatively and discharged on a reduced dose of imatinib $200 \mathrm{mg}$ daily. 
Upon consultation, the primary surgeon recommended resection of the new mass and the plan was to have a discussion with the patient about a possible resection. However, after discharge, the patient's condition continued to deteriorate and she returned eight days later complaining of nausea, difficulty retaining food, ongoing abdominal discomfort, dizziness and weakness. The patient also noted that her urine was getting darker and felt she was urinating less than usual. She denied any pyuria, dysuria, nocturia, hematuria, fever or chills. The patient's medication history was negative for non-steroidal anti-inflammatory medications and nephrotoxic drugs. The patient, despite having nausea, continued to take her imatinib as prescribed at the reduced dose. The medication was stopped upon admission.

On physical examination the patient's abdomen was soft and distended with diffuse tenderness on palpation, but no guarding or shifting dullness. A large palpable mass could be felt in the periumblical region. The patient subsequently underwent a repeat CT scan of her abdomen and pelvis without contrast, which showed an increase in the size of the mass and enlarged diaphragmatic lymph nodes. Laboratory evaluation upon admission showed a blood urea nitrogen (BUN) of $7.8 \mathrm{mmol} / \mathrm{L}(\mathrm{N}: 3-7 \mathrm{mmol} / \mathrm{L})$ and a creatinine of 422 umol/L (N: 50-98 umol/L), elevated from her baseline of $79.2 \mathrm{umol} / \mathrm{L}$. Serum calcium was $1.96 \mathrm{mmol} / \mathrm{L}$ $(\mathrm{N}: 2.2-2.5 \mathrm{mmol} / \mathrm{L})$, phosphorous $0.65 \mathrm{mmol} / \mathrm{L}(\mathrm{N}$ : 1.0-1.5 mmol/L) and potassium $4.4 \mathrm{mmol} / \mathrm{L}(\mathrm{N}: 3.5-5.0$ $\mathrm{mmol} / \mathrm{L}$ ). Urine analysis was normal with no white or red blood cells, or visible urine casts. Renal ultrasound did not show evidence of obstruction. Nephrology was consulted and an initial diagnosis of pre-renal azotemia and possible acute tubular necrosis secondary to dehydration and renal hypo-perfusion was made. The patient was started on intravenous hydration with close monitoring of her urine output.

The following day, the patient was still found to be oliguric. A repeat basic metabolic panel revealed worsening renal function, with an elevation of serum creatinine to $512 \mathrm{umol} / \mathrm{L}, \mathrm{BUN}$ of $38.5 \mathrm{mmol} / \mathrm{L}$, potassium of $5.0 \mathrm{mmol} / \mathrm{L}$, calcium of $1.82 \mathrm{mmol} / \mathrm{L}$, phosphorous of $1.19 \mathrm{mmol} / \mathrm{L}$ and an anion gap of 16 . Uric acid was checked and found to be $2218 \mathrm{umol} / \mathrm{L}$, at which point the patient was given rasburicase 6 mg intravenously and allopurinol $100 \mathrm{mg}$ PO daily as per renal dosing. A vas-cath was placed urgently and the patient was started on hemodialysis. Despite improvement in her metabolic derangements, the patient continued to experience persistent nausea and vomiting. In view of her poor prognosis and poor performance status, the patient and the family decided to pursue comfort measures and palliative care. The patient passed away within twenty four hours.

\section{Discussion}

TLS is an oncological emergency caused by massive tumor cell lysis and subsequent release of intracellular metabolites in to the blood stream, leading to a constellation of metabolic derangements. The associated increase in plasma contents of potassium, uric acid and phosphorous, in particular, can overwhelm the kidneys capacity to clear these substances leading to hyperkalemia, hyperphosphatemia, and hyperuricemia potentially causing serious and life threatening complications. The resulting hyperkalemia, if left untreated, can lead to lethal cardiac arrhythmias. The buildup of uric acid and elevated phosphate can lead to calcium phosphate precipitate forming stones in the nephron tubules causing renal vasoconstriction, impaired auto regulation, and decreased perfusion to the kidneys leading to acute kidney injury. In addition, secondary hypocalcemia caused by binding of calcium to excess phosphorous in the blood stream can lead to neuromuscular irritability. As a result of these metabolic derangements, patients can present with a constellation of symptoms including nausea, vomiting, diarrhea, anorexia, lethargy, seizures, muscle cramps, and an increased risk of sudden death.

Historically, TLS occurs after treating hematologic malignancies such as leukemias (e.g. Acute Lymphoblastic Leukemia) and lymphomas (particularly the Burkitt subtype). ${ }^{1-3}$ However, TLS can also occur spontaneously or after medical interventions such as radiation, immunotherapy, targeted therapies or other endocrine therapies that lead to tumor cell death. ${ }^{1-3}$ TLS rarely occurs in solid tumors, but if it does, it is usually associated with more advanced bulky solid tumors with high proliferative rates, tumors with high sensitivity to chemotherapeutic agents, or more uncommonly, it can be associated with embolization of liver metastasis. ${ }^{1-3}$

GIST is a solid tumor that is not well recognized to cause the development of TLS. GIST, although rare, is the most common non-epithelial tumor of the gastrointestinal tract. In the past the mortality rate for patients with GIST was high because of minimal response to chemotherapeutic agents. ${ }^{4,5}$ However, the introduction of tyrosine kinase inhibitors, such as imatinib mesylate, that target the extracellular protein KIT (CD117) expressed on GISTs have led 
to a rapid improvement in clinical response. Since the introduction of imatinib, the mean survival has increased from 18 to 57 months. As a result, imatinib is now considered the standard first line treatment for advanced unresectable GIST. ${ }^{4,5}$

Although imatinib has significantly improved mortality in patients with GIST, it can be associated with significant side effects. Known non life-threatening side effects of Imatinib include fluid retention, muscle cramps, gastrointestinal disturbance (e.g., nausea and vomiting), rash, mild anemia, elevated liver function tests, and heart failure in those with pre-existing heart disease. ${ }^{4,5}$ Uncommon side effects include neutropenia and tumor hemorrhage. One of the most severe complications associated with imatinib is TLS.

Although there have been case reports of TLS in patients with Chronic Myelogenous Leukemia receiving imatinib, it is almost never heard of in GIST. ${ }^{6}$ To our knowledge, there has only been one case report in the literature showing TLS after imatinib therapy. ${ }^{7}$ This was associated with a metastatic aggressive GIST subtype. This patient not only had a bulky aggressive tumor, but also had gout, dehydration and was on nephrotoxic drugs that enhanced his risk for TLS. The typical starting dose for imatinib is $400 \mathrm{mg} /$ day, however a dose of $800 \mathrm{mg} /$ day is commonly used when patients have a KIT exon 9 mutation or when patients stop responding to the standard $400 \mathrm{mg} /$ day dose. ${ }^{8}$ This case report, as well as the one in 2007, demonstrated TLS occurring at the smaller dose of $400 \mathrm{mg}$. Unfortunately, both patients died as a consequence of TLS.

As TLS is a serious and potentially fatal complication, early recognition of risk factors and prevention are essential to appropriate management. Prognostic factors that help predict the risk of TLS include dehydration, baseline renal dysfunction, hyperuricemia and hyperphosphatemia. ${ }^{9}$ Currently, the best preventative measure is prophylactic intravenous hydration to enhance clearance of toxic metabolites, improve renal perfusion and glomerular filtration, and increase urine output to minimize the likelihood of uric acid or calcium phosphate precipitation in nephron tubules. ${ }^{9}$ As prevention is the best measure, two drugs that are commonly used and work well to lower uric acid are rasburicase and allopurinol. Although both medications are effective, rasburicase is generally preferred because of its more rapid onset and preferred mechanism of action. Unlike allopurinol, which is a competitive inhibitor of xanthine oxidase and can lead to accumulation of purine precursors and cause acute kidney injury, rasburicase is a recombinant uric oxidase that catalyzes oxidation of uric acid to a more watersoluble compound allantion. In the setting of TLS, rasburicase may be a safer and more effective option because it decreases preexisting levels of uric acid, leads to a more rapid reduction in serum uric acid and does not lead to xanthine accumulation. ${ }^{10}$

\section{Conclusion}

It is important for clinicians to recognize that TLS is a rare, life threatening complication of cancer treatment that can occur in many different types of cancers. It is also important to note that new biologically directed agents for cancer treatments can lead to TLS. Special attention should be placed on risk factors that can potentially lead to TLS, which include: poor hydration, inadequate renal function, high tumor burden and elevated levels of lactate dehydrogenase. Patients with these specific high risk factors may be considered for prophylactic hydration for TLS prevention.

\section{Acknowledgements}

Dr. Ajeet Gajra at SUNY Upstate Medical University in Syracuse NY and Dr.Don Morris at the Tom Baker Cancer center in Calgary Alberta.

\section{References}

1. Hochberg J, Cairo MS. Tumor lysis syndrome: current perspective. Haematologica 2008; 93(1):9-13.

2. Davidson MB, Thakkar S, Hix JK, Bhandarkar ND, Wong A, Schreiber MJ. Pathophysiology, clinical consequences, and treatment of tumor lysis syndrome. Am J Med 2004;116(8):546-54.

3. Darmon M, Guichard I, Vincent F, Schlemmer B, Azoulay E. Prognostic significance of acute renal injury in acute tumor lysis syndrome. Leuk Lymphoma 2010;51(2):221-7.

4. Ksienski D. Imatinib Mesylate: Past successes and future challenges in the treatment of GIST. Clin Med Insights Oncol 2011;5:365-79.

5. Joensuu H, Roberts PJ, Sarlomo-Rikala M, Andersson LC, Tervahartiala P, Tuveson D, et al. Effect of the tyrosine kinase inhibitor STI571 in a patient with a metastatic gastrointestinal stromal tumor. N Engl J Med 2001;344(14):1052.

6. Al-Kali A, Farooq S, Tfayli A. Tumor lysis syndrome after starting treatment with Gleevec in a patient with chronic myelogenous leukemia. J Clin Pharm Ther 2009;34(5):607-10.

7. Pinder EM, Atwal GS, Ayantunde AA, Khan S, Sokal M, McCulloch T, et al. Tumour lysis syndrome occcurring in a patient with metastatic gastrointestinal stromal tumour treated with Glivec (Imatinib Mesylate, Gleevec, STI571). Sarcoma 2007;2007:82012.

8. Blanke C, Rankin C, Demetri G, Ryan C, Mehren M, Benjamin R, et al. Phase III randomized, intergroup trial assessing imatinib mesylate at two dose levels in patients with unresectable or metastatic gastrointestinal stromal tumors expressing the kit receptor tyrosine kinase: S0033. J Clin Oncol 2008;26(4):626-32.

9. Coiffier B, Altman A, Pui CH, Younes A, Cairo MS. Guidelines for the management of pediatric and adult tumor lysis syndrome: an evidencebased review. J Clin Oncol 2008;26(16):2767-78.

10. Bose P, Qubaiah O. A review of tumor lysis syndrome with targeted therapies and the role of rasburicase. J Clin Pharm Ther 2011;36(3):299326 